\title{
Standing Stability in Knee Osteoarthritis Patients
}

\author{
Karimi M.T. ${ }^{1}$ PhD, Esrafilian A. ${ }^{2}$ PhD, Salahi M.* BSc
}

*Technical Orthopedic Department, Rehabilitation Sciences Faculty, Isfahan University of Medical Sciences, Isfahan, Iran

${ }^{1}$ Technical Orthopedic Department, Rehabilitation Sciences Faculty, Isfahan University of Medical Sciences, Isfahan, Iran

2Department of Advanced Technologies in Medicine, Biomedical Engineering Faculty, Amirkabir University of Technology, Tehran, Iran

\begin{abstract}
Aims: Being prevalent in the more than 40 years old persons, the knee osteoarthritis is one of the main factors in the function system failure mainly affecting their life. There is a type of instability in the persons with knee osteoarthritis, which is an increase in the domain and frequency of body pressure center. The aim of the present study was to compare the standing stability parameters in persons with knee osteoarthritis and healthy persons.

Instrument $\&$ Methods: In this case-control, 15 patients with knee osteoarthritis referred to the physiotherapy clinic of the rehabilitation center and Al-Zahra hospital were studied in the muscle-skeletal research center of Isfahan University of Medical Sciences in 2015. The subjects were selected via stratified sampling method. As control group, 15 healthy persons were also studied in Isfahan. Total path length and the domain and frequency of pressure center in different directions were measured to assess the stability. Data was analyzed by SPSS 19 software using descriptive statistics and paired T test.

Findings: There were increases in the mean movement domain and the frequency of pressure center in the anterior-posterior and the internal-external directions, as well as in the total path proceeded by the pressure center in the internal-external direction, in the persons with knee osteoarthritis than the healthy persons $(\mathrm{p}<0.05)$. Nevertheless, there was no significant difference in the mean total path proceeded by the pressure center in the anterior-posterior direction ( $\mathrm{p}>0.05)$.

Conclusion: Standing stability and balance in persons with knee osteoarthritis decreases compared to healthy persons.

\section{Keywords}

Static Stability [Not in MeSH];

Osteoarthritis, Knee [http://www.ncbi.nlm.nih.gov/mesh/68020370];

Force Plate [Not in MeSH]

\footnotetext{
${ }^{*}$ Corresponding Author

Tel: +985138213461

Fax: -

Address: Technical Orthopedic Department, Rehabilitation Sciences Faculty, Hezar Jarib Street, Isfahan University of Medical Sciences, Isfahan, Iran. Postal Code: 81746-73461

mohammadsalahi68@yahoo.com

Received: August 24, $2015 \quad$ Accepted: February 9, 2016 ePublished: June 30, 2016
} 
فشار خون غيرمتعارف يا حتى ديابت بيشتر است[1]. اين عارضه

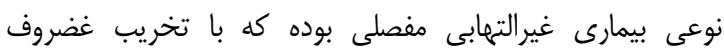

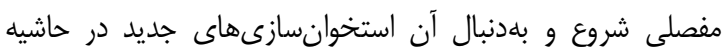

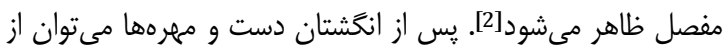

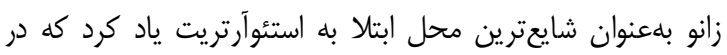

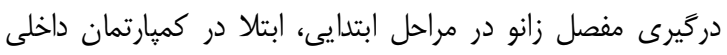

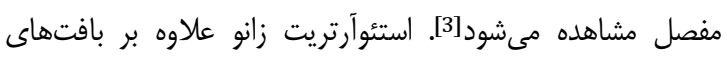

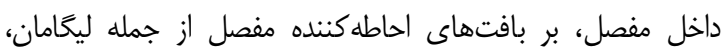

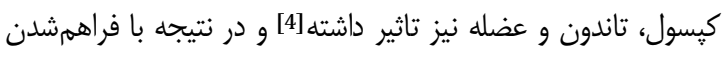

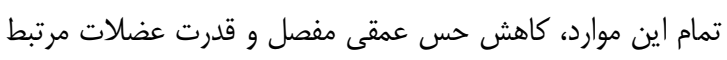

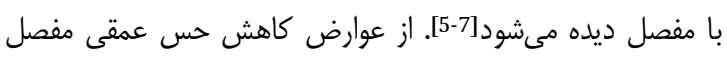

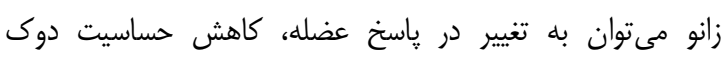

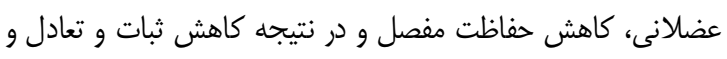

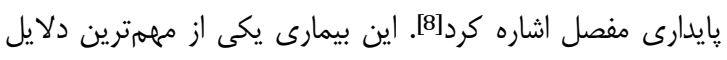

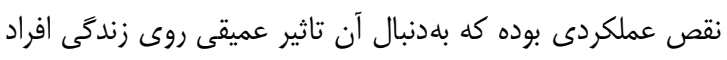

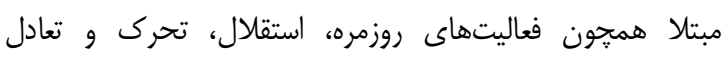

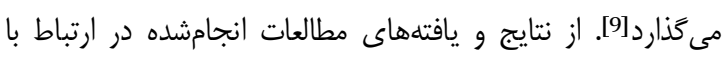

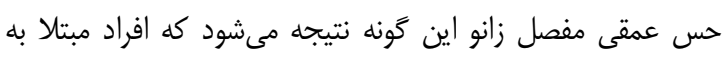

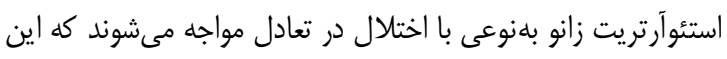

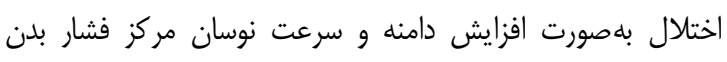

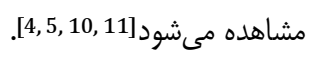
اخيراً از صفحه نيرو براى بررسى و آناليز ميزان و سرعت نوسان

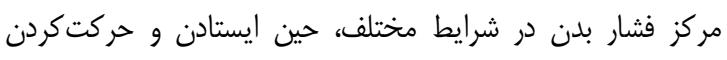

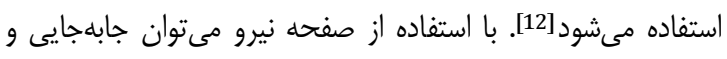

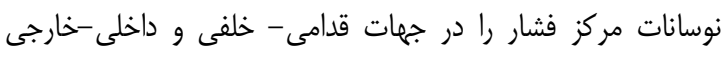

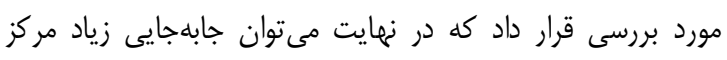

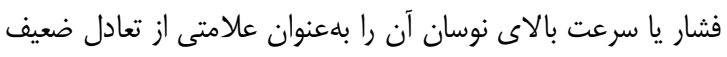

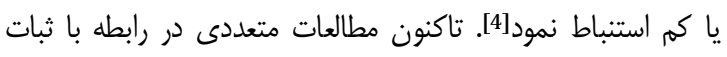

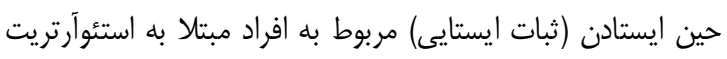

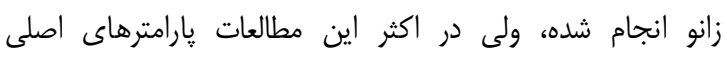

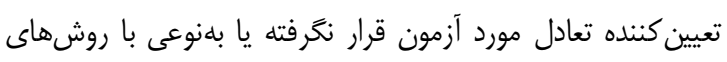

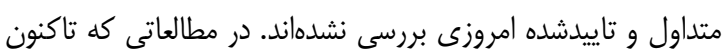

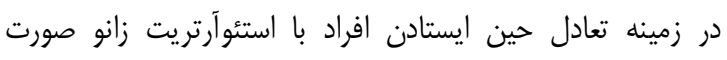

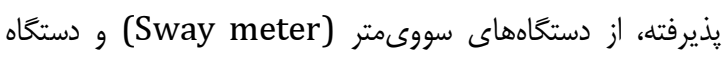

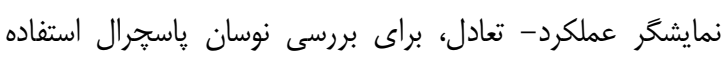

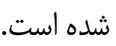
حسان و همكاران با استفاده از دستخاه نمايشكر عملكرد- تعادل،

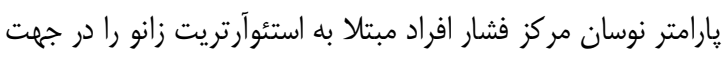

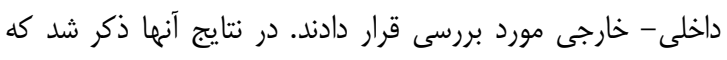

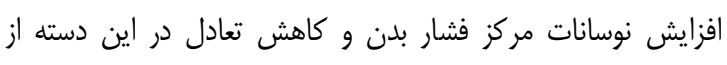

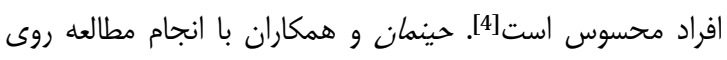

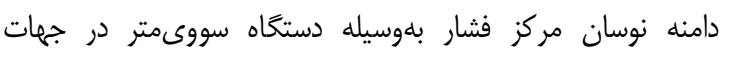

\section{ثبات ايستادن در مبتلايان به استئوآرتريت زانو}

محمدتقى كريمى PhD

كروه ارتويدى فنى، دانشكده علوم توانبخشى، دانشگاه علوم يزشكى اصفهان،

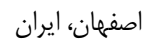

PhD امير اسرافيليان إنان

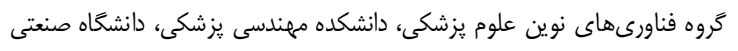
امير كبير، تهران، ايران

BSc محمد صلاحى كروه ارتويدى فنى، دانشكده علوم توانبخشى، دانشگاه علوم يزشكى اصفهان، اصفهان، ايران

جکكيده

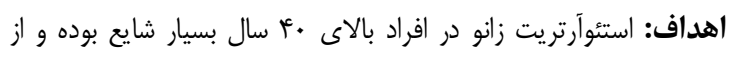

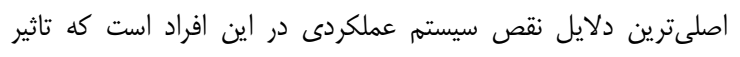

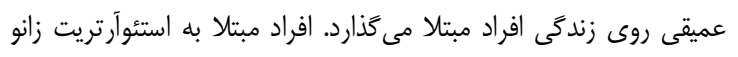

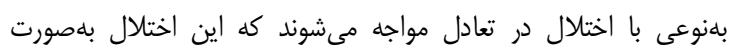

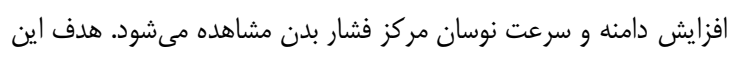

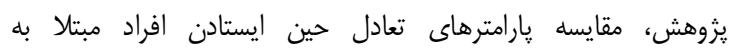
استئوآرتريت زانو با افراد سالم بود.

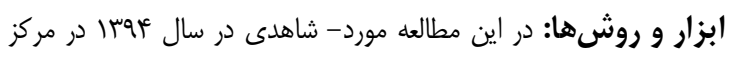

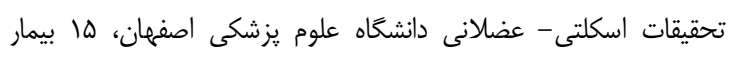

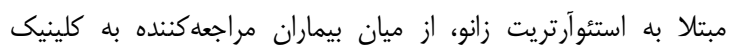

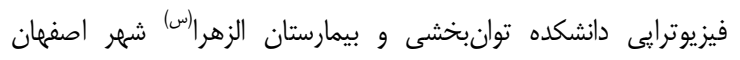

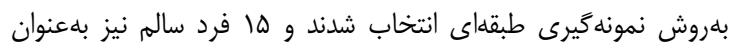

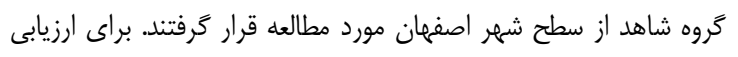

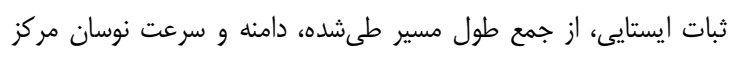

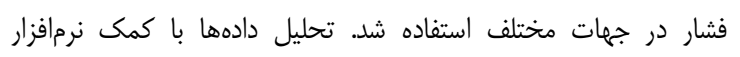

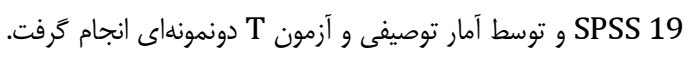

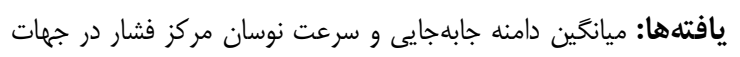

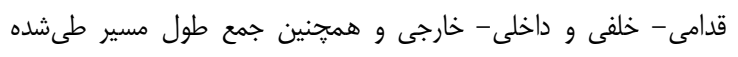

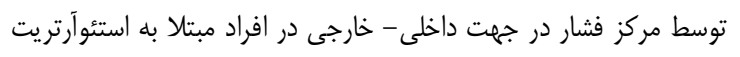

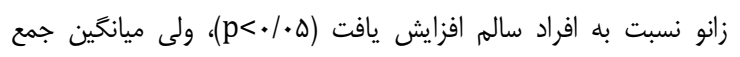

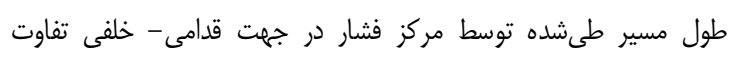

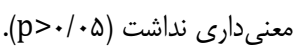
نتيجه كيرى: ثبات و تعادل حين ايستادن در افراد مبتلا به استئوآرتريت زانو نسبت به افراد سالم كاهش ييدا مئ كندي

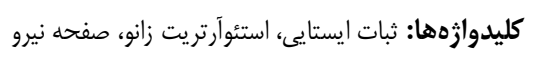

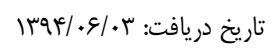

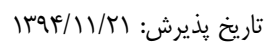
mohammadsalahi68@yahoo.com : تويسنده مسئول: تاريخ:

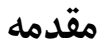

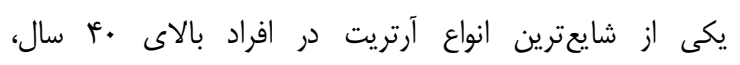

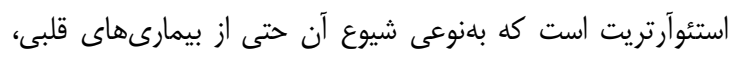

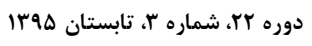

فصلنامه افق دانش 
ثبات ايستادن در مبتلايان به استئو آرتريت زانو هائ

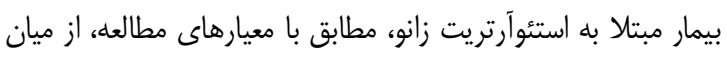

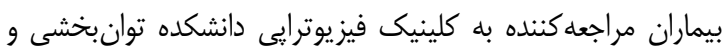

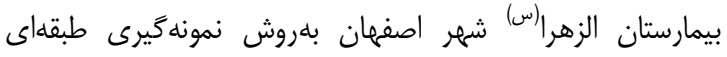

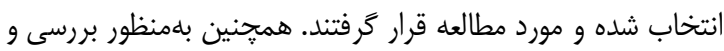

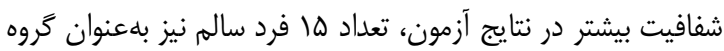
شاهد از سطح شهر اصفهان انتخاب شدند.

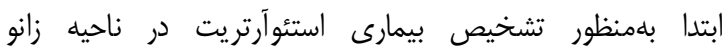

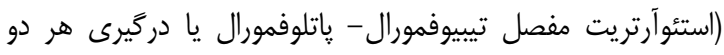

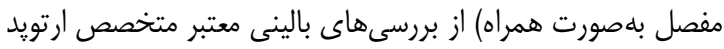

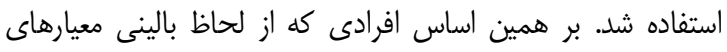

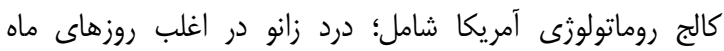

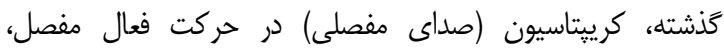

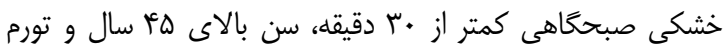

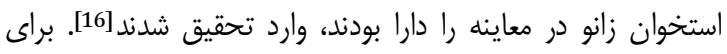

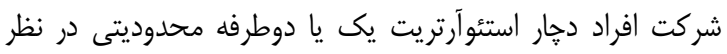

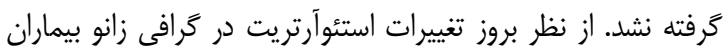

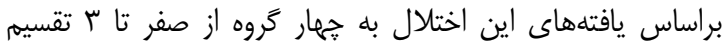

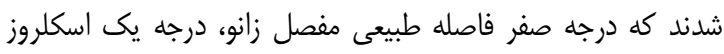

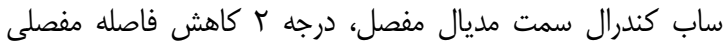

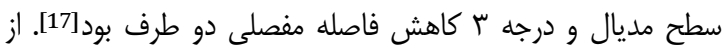

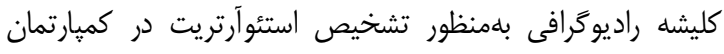

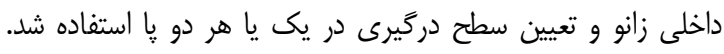

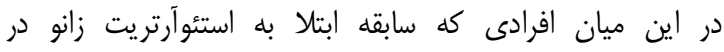

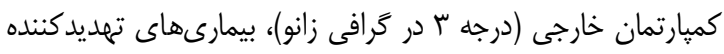

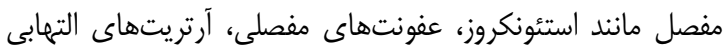

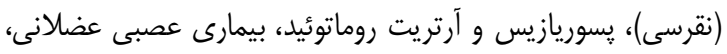

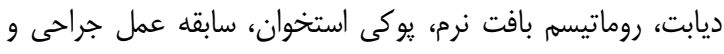

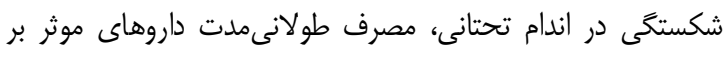

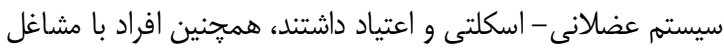

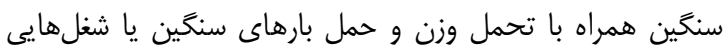

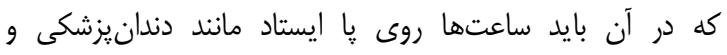

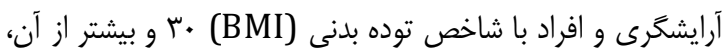

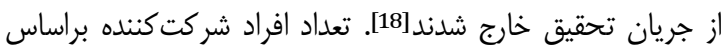

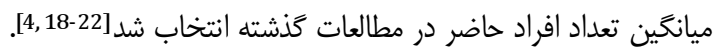

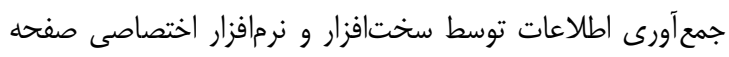

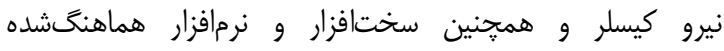

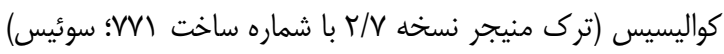

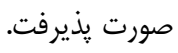

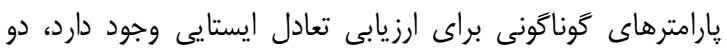

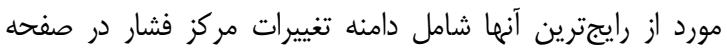

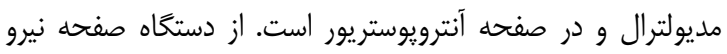

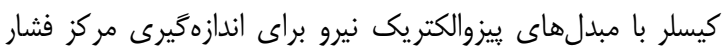

داخلى - خارجى و قدامى - خلفى در افراد مبتلا به استئوآرتريت زانو

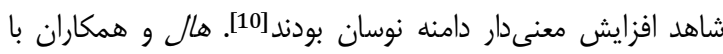

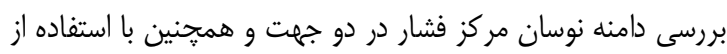

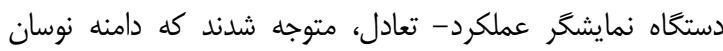

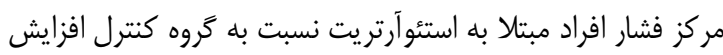

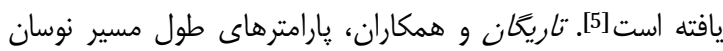

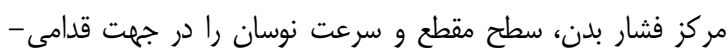

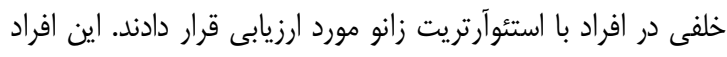

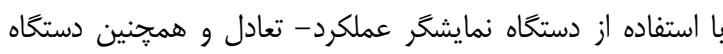

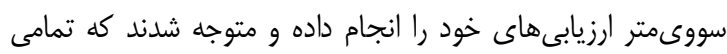

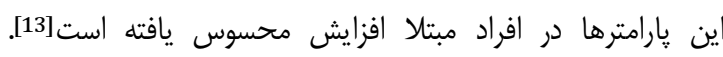

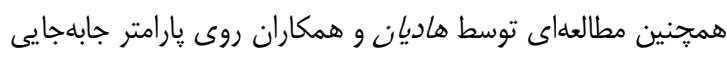

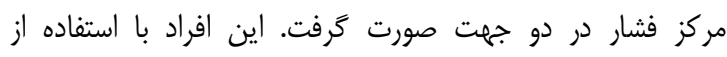

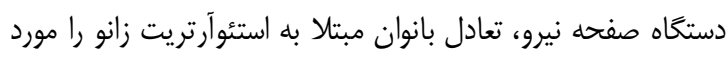

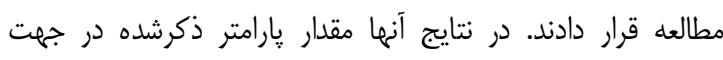

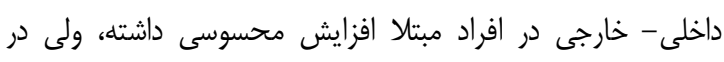

$$
\text { جهت قدامى - خلفى افزايش معنى دار نبود [14]. }
$$

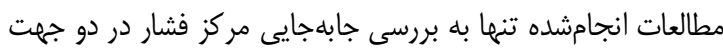

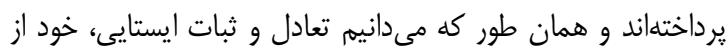

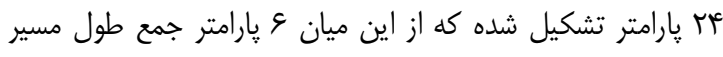

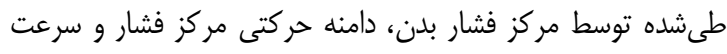
نوسان مركز فشار در جهات قدامى - خلفى و داخلى - خارجى قابليت دركيت اطمينان بيشترى براى بررسى تعادل دارند [15.].

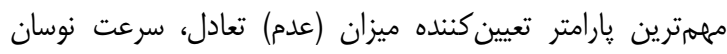

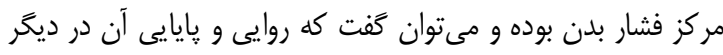

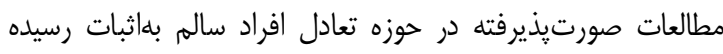

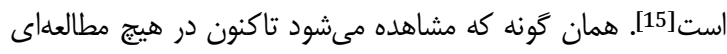

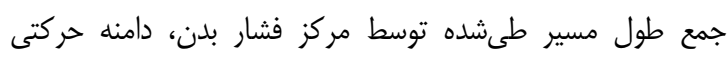

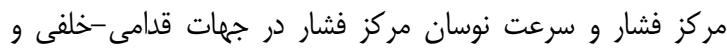

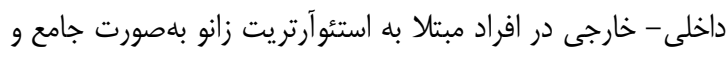

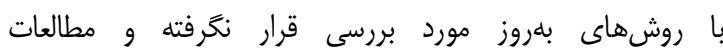

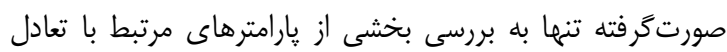
افراد مبتلا ير داختهاند.

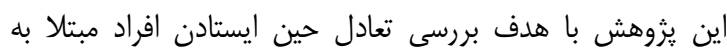

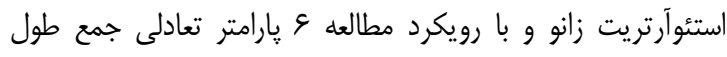

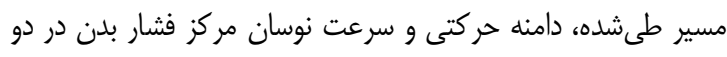
جهت مختلف در اين گروه از افراد و مقايسه آن با افراد سالم انجام فرام

\section{ابزار و روشها}

در اين مطالعه مورد- شاهدى كه در سال عاوسا در مركز تحقيقات

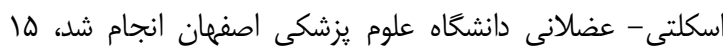


جدول () ويثَى هاى دموكَرافيك شركت كنندًان سالم و افراد مبتلا به استئو آرتريت

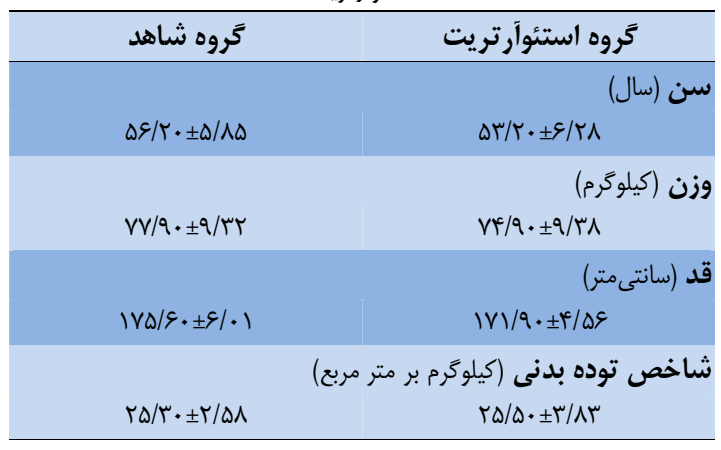

اختلاف ميانكين يارامتر جمع طول مسير طىشده توسط مركز فشار

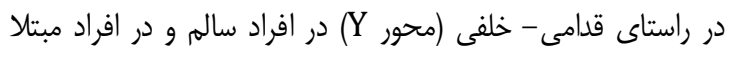

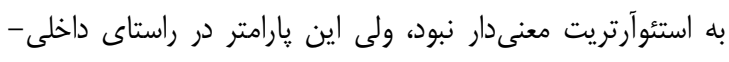

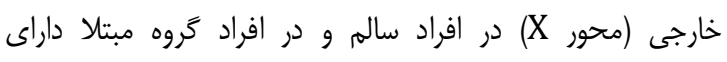

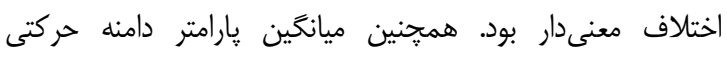

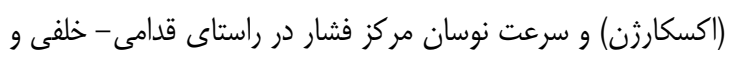

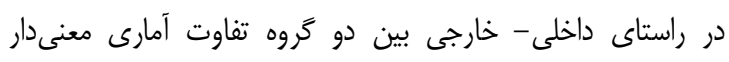

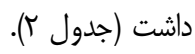

جدول T) مقايسه ميانكين آمارى يارامترهاى تعادلى در رابطه با مركز فشار بدن در دو گروه

\begin{tabular}{|c|c|c|c|}
\hline سطح معنىدارى & استئوآرتريت & شاهد & يارامترها \\
\hline \multirow{3}{*}{$\begin{array}{l}1 \cdot \wedge \mu \\
. \ldots \mid r\end{array}$} & \multicolumn{3}{|c|}{ جمع طول مسير (ميلىمتر) } \\
\hline & $\mid V r J / \cdot V \pm G T / / I I$ & $9 \varphi 9 / V \wedge \pm q r / \uparrow \wedge$ & قدامى - خلفى \\
\hline & $10 \Delta N / V q \pm 99 \cdot / 49$ & SNN/DVIIFV/GY & داخلى - خارجى \\
\hline & & (ميلى متر) & دامنه حركتى ( \\
\hline $.1 .4 \wedge$ & $r q / v \wedge \pm r q / r \wedge$ & $r \cdot / 1 \cdot \pm 14 / 4 r$ & قدامى - خلفى \\
\hline 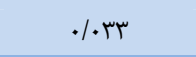 & $r \Psi / \cdot V \pm V / l$. & $\mid v / 19 \pm q / \cdot v$ & داخلى - خارجى \\
\hline & & إ (ميلىمتر بر ثانيه) & سرعت نوساز \\
\hline $.1 \cdots 10$ & $8 / \Delta \cdot \pm 1 / r q$ & $r / V I \pm \cdot / \Delta F$ & قدامى - خلفى \\
\hline 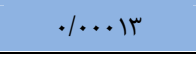 & $8 / \pi \pm 1 / \pi T^{\circ}$ & $T / V \Delta \pm \cdot / \Delta \Delta$ & داخلى - خارجى \\
\hline
\end{tabular}

بحث

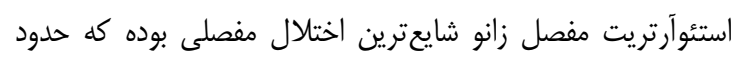

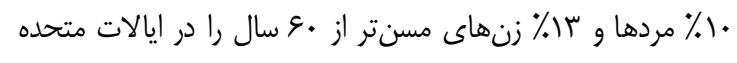

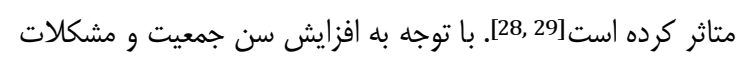

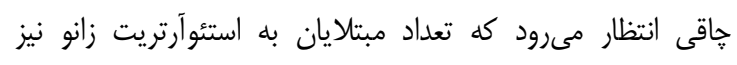

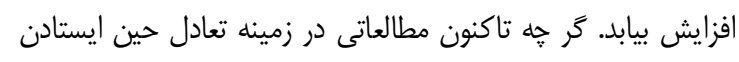

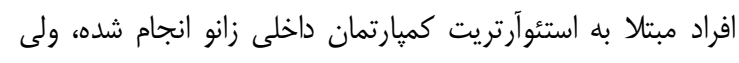

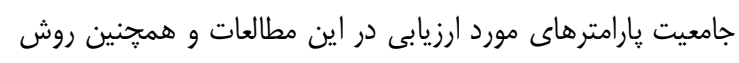

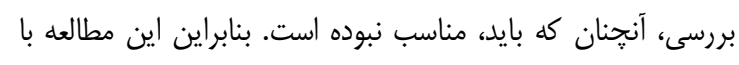

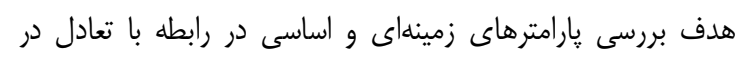

كه بهعنوان تقريب خوبى از نوسارن است است استفاده مى شودد. نوسان در

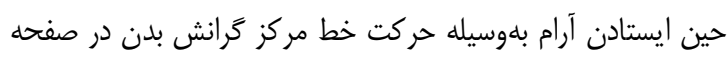

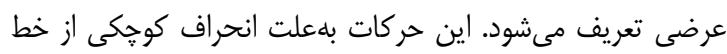

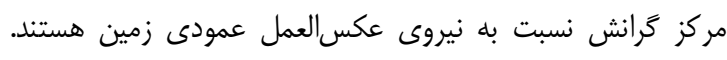

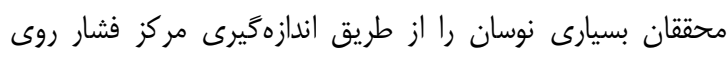

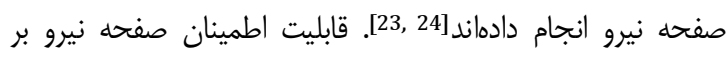

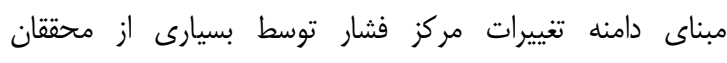

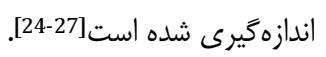

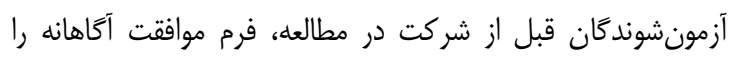

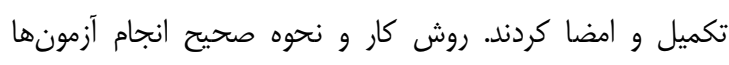

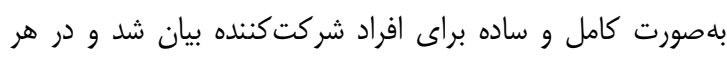

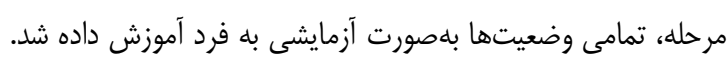

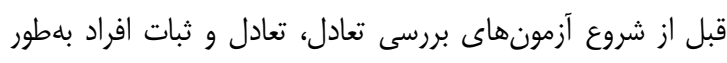

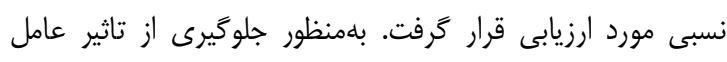

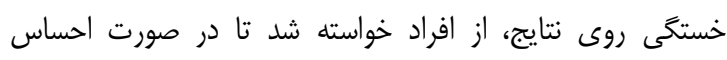

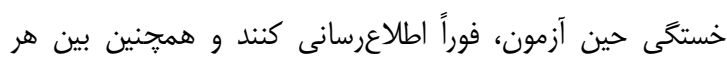

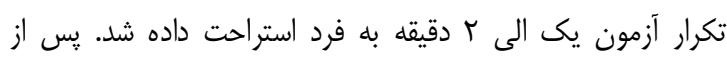

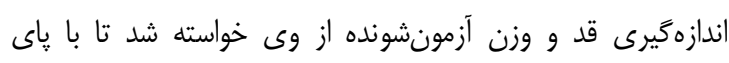

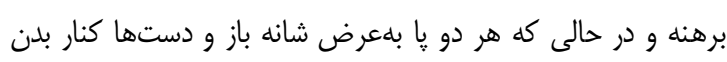

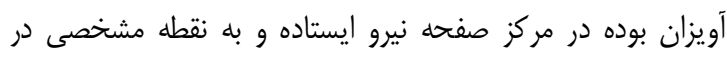

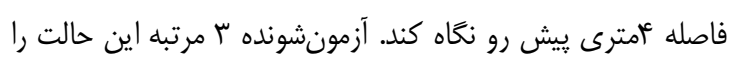

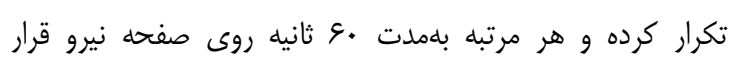

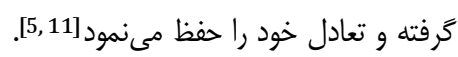

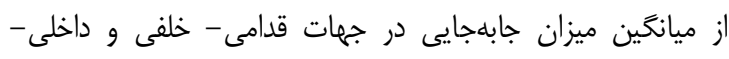

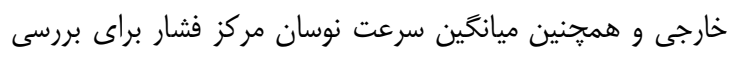

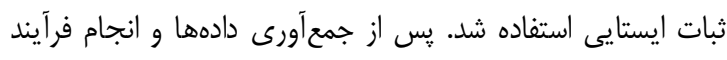

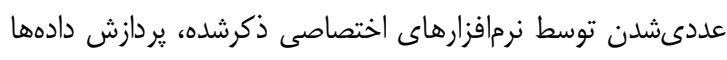

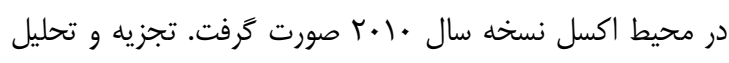

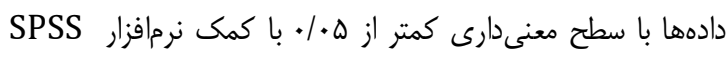

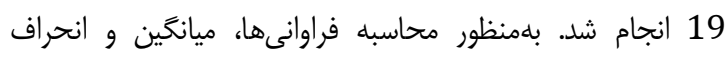

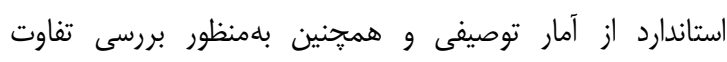

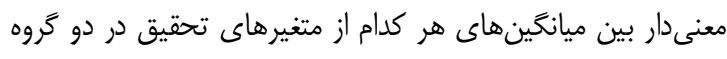

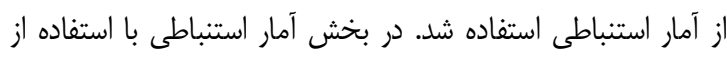

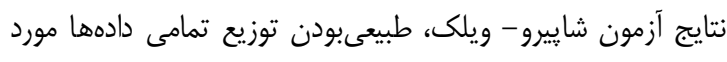

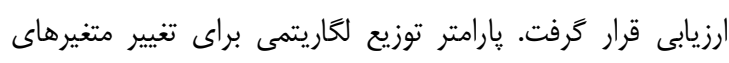

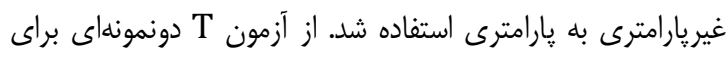

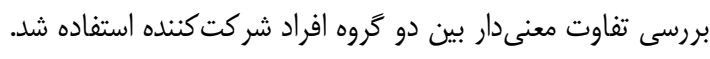

\section{يافتهها}

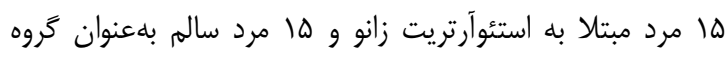

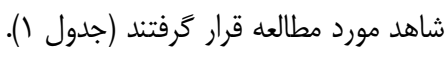




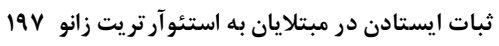

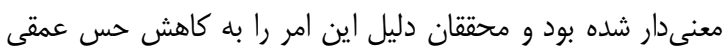

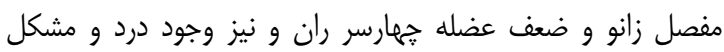

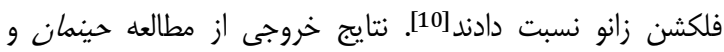

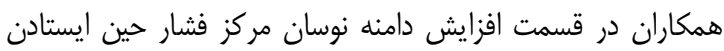

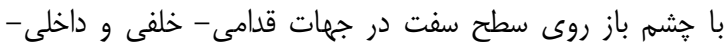

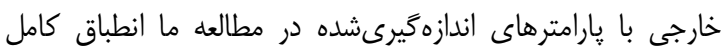

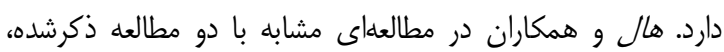

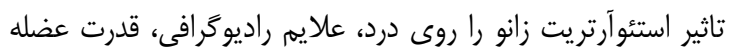

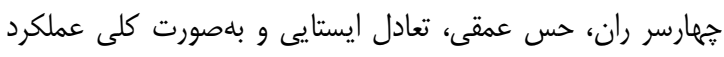

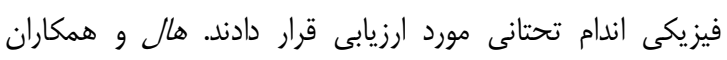

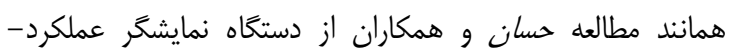

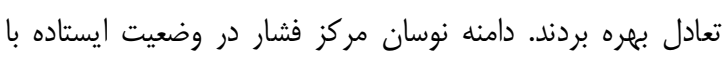

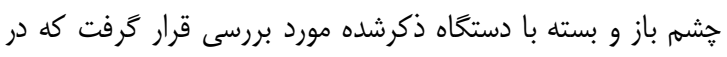

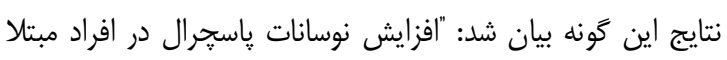

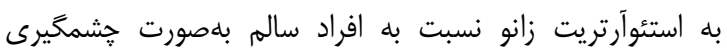

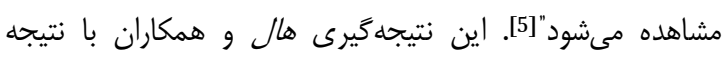

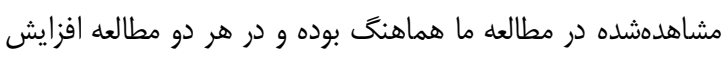
دامنه نوسان پاسجر و مركز فشار كه تقريب نسبتاً مناسبى از مركز

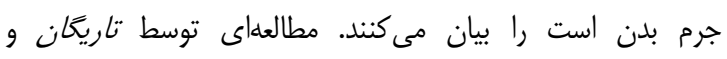

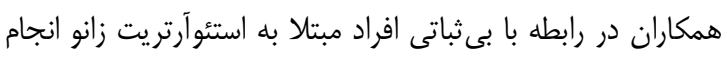

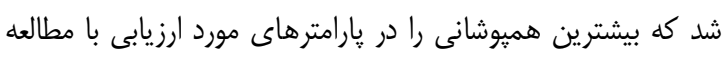

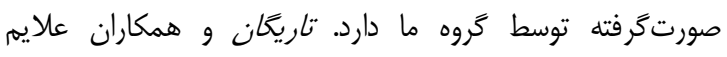

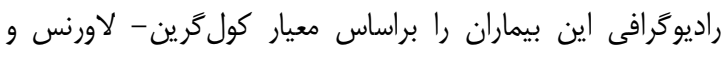

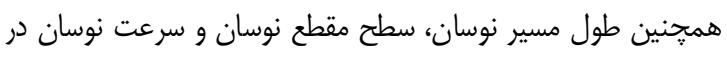

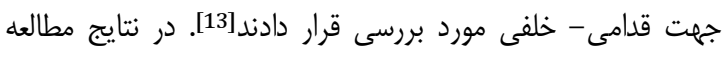

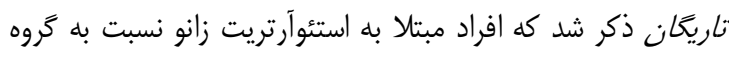

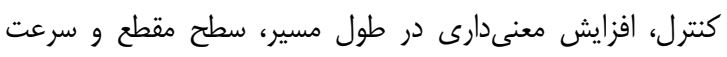

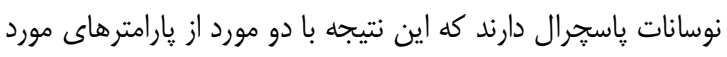

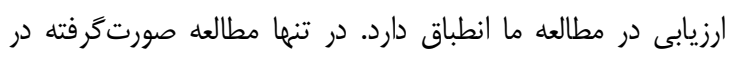

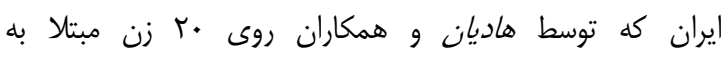

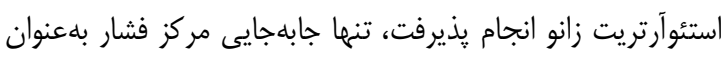

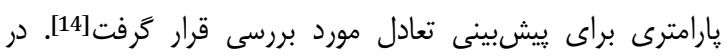

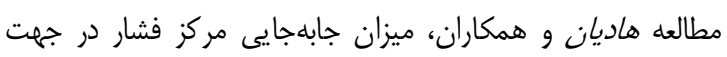

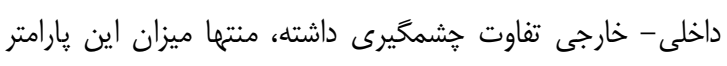

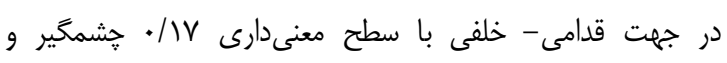

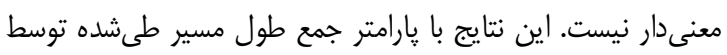

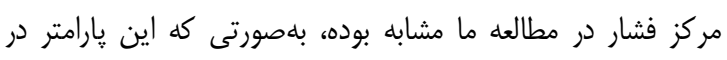

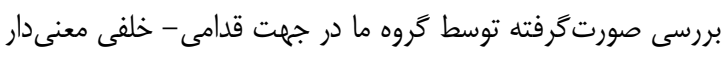

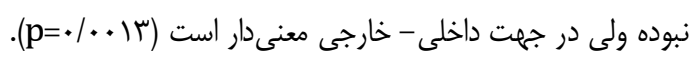

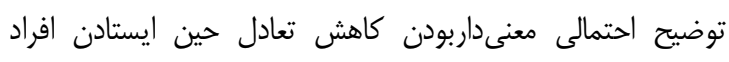

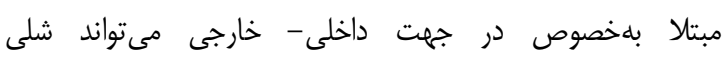

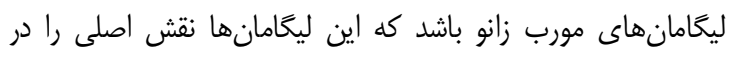

افراد مبتلا به استئوآرتريت زانو با جامعه قابل قبولى از افراد مبتلا

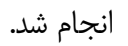

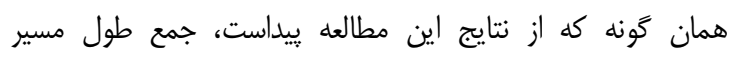

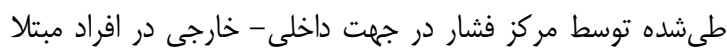

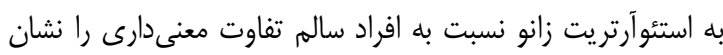

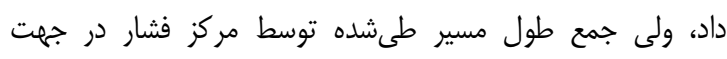

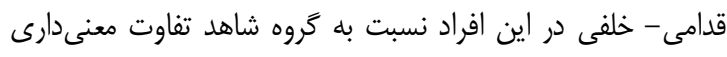

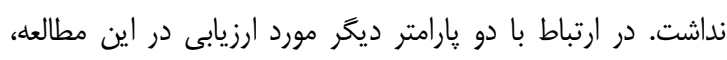

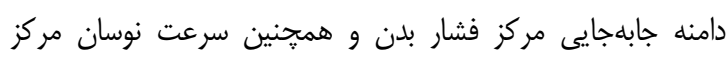

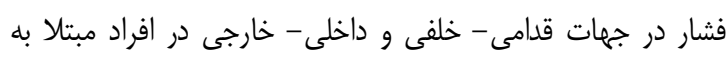

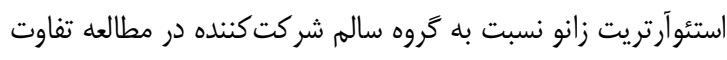

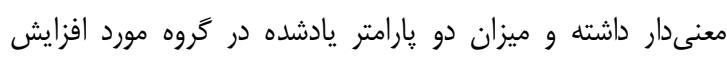

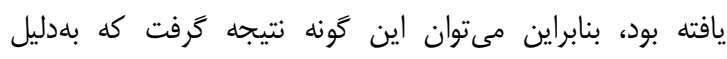

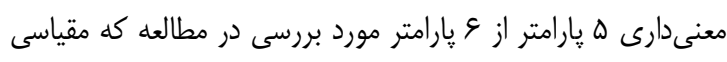

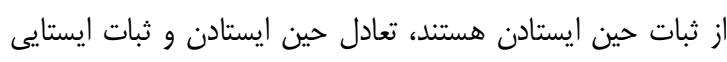

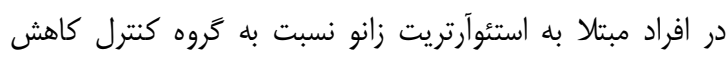

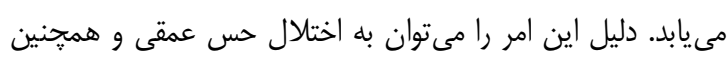

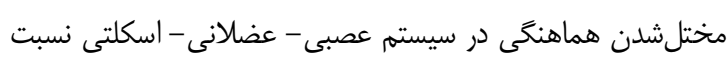

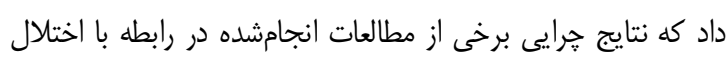

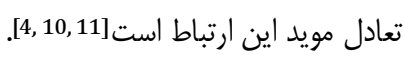

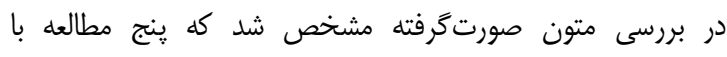

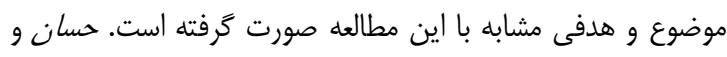

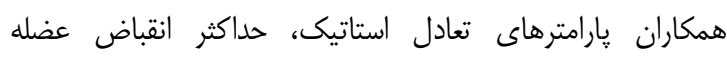

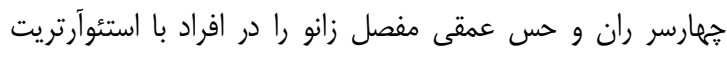

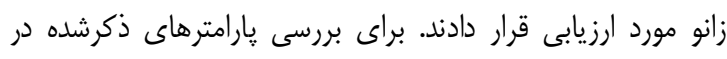

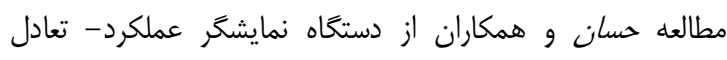

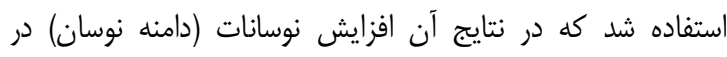

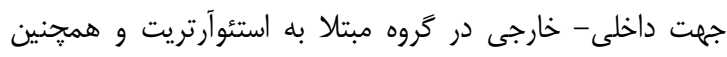

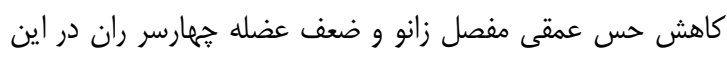

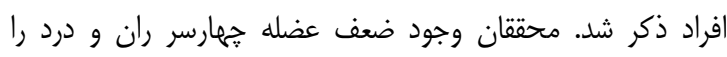

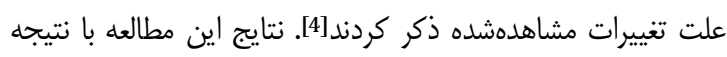

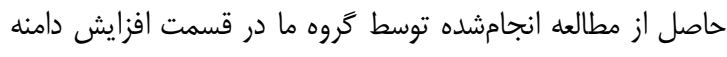

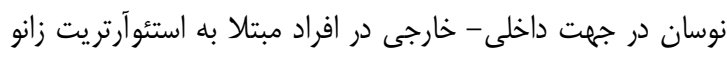

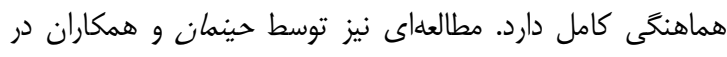

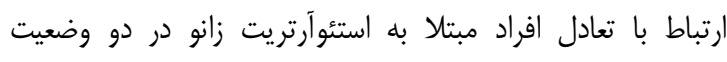

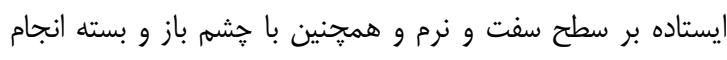

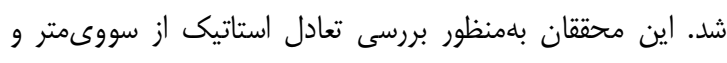

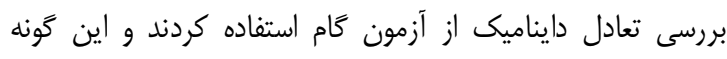

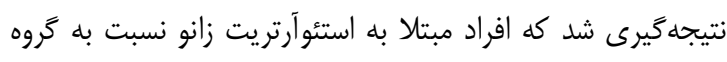

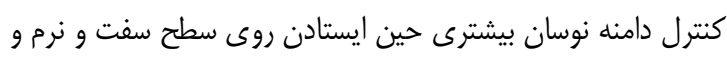

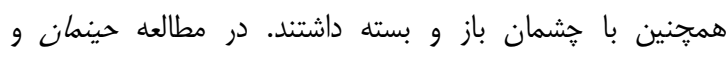

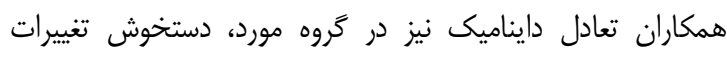


مطالعه يارى فرمودند، كمال تشكر و قدردانى را داريم.

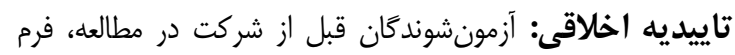

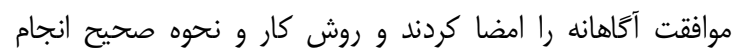

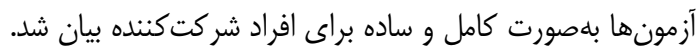
تعارض منافع: هيج كَونه تعارض منافع توسط نويسندكان بيان نشده است.

منابع مالى: اين مقاله حاصل طرح تحقيقاتى مصوب (كد:

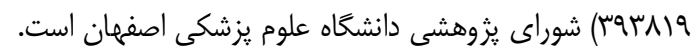

منابع

1- Kelsey JL, MC Hochberg. Epidemiology of chronic musculoskeletal disorders. Annu Rev Public Health. 1988;9:379-401.

2- Martel-Pelletier J, Lajeunesse D, Pelletier JP. Etiopathogenesis of osteoarthritis. In: Koopman WJ, editor. Arthritis and allied conditions: A textbook of rheumatology. 15th edition. Baltimore, USA: Lippincott, Williams \& Wilkins; 2005. pp. 2199-226.

3- Klippel JH, Stone JH, Crofford LJ, White PH. Primer on the rheumatic diseases. Verlag New York: Springer Science \& Business Media; 2008.

4- Hassan B, Mockett M, Doherty M. Static postural sway, proprioception, and maximal voluntary quadriceps contraction in patients with knee osteoarthritis and normal control subjects. Ann Rheum Dis. 2001;60(6):612-8.

5- Hall MC, Mockett SP, Doherty M. Relative impact of radiographic osteoarthritis and pain on quadriceps strength, proprioception, static postural sway and lower limb function. Ann Rheum Dis. 2006;65(7):865-70.

6- Sharma L. Proprioceptive impairment in knee osteoarthritis. Rheum Dis Clin North Am. 1999;25(2):299-314.

7- Sharma L, Pai YC. Impaired proprioception and osteoarthritis. Curr Opin Rheumatol. 1997;9(3):253-8.

8- Garsden L, Bullock-Saxton J. Joint reposition sense in subjects with unilateral osteoarthritis of the knee. Clin Rehabil. 1999;13(2):148-55.

9- Salavati M, Mazaheri M, Negahban H, Sohani SM, Ebrahimian MR, Ebrahimi I, et al. Validation of a Persianversion of knee injury and osteoarthritis outcome score (KOOS) in Iranians with knee injuries. Osteoarthritis Cartilage. 2008;16(10):1178-82.

10- Hinman RS, Bennell KL, Metcalf BR, Crossley KM. Balance impairments in individuals with symptomatic knee osteoarthritis: a comparison with matched controls using clinical tests. Rheumatol. 2002;41(12):1388-94.

11- Masui T, Hasegawa Y, Yamaguchi J, Kanoh T, Ishiguro $\mathrm{N}$, Suzuki S. Increasing postural sway in ruralcommunity-dwelling elderly persons with knee osteoarthritis. J Orthopaedic Sci. 2006;11(4):353-8.

12- Hughes MA, Duncan PW, Rose DK, Chandler JM, Studenski SA. The relationship of postural sway to sensorimotor function, functional performance, and disability in the elderly. Arch Phys Med Rehabil. 1996;77(6):567-2.

13- Tarigan TJ, Kasjmir YI, Atmakusuma D, Lydia A, Bashiruddin J, Kusumawijaya $\mathrm{K}$, et al. The degree of radiographic abnormalities and postural instability in patients with knee osteoarthritis. Acta Med Indones. 2009;41(1):15-9.

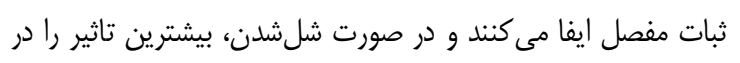

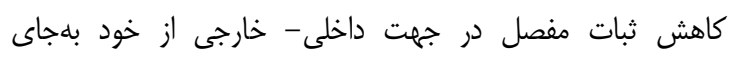

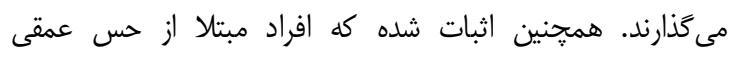

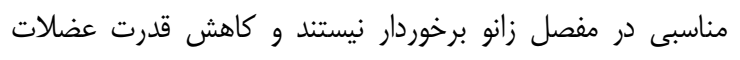

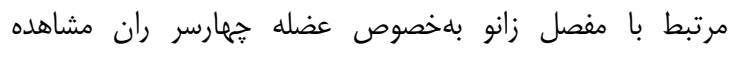

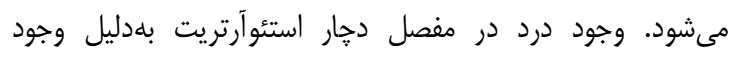

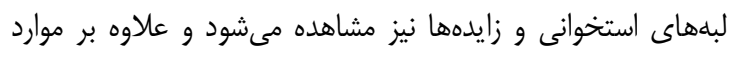

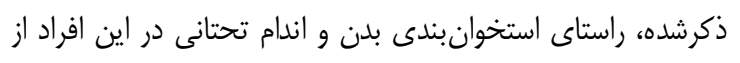

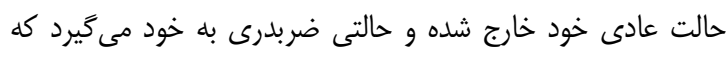

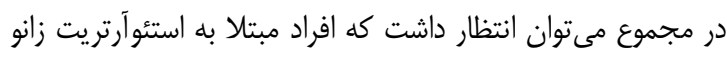

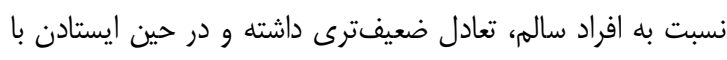
جابهجايى زياد مركز فشار بدن مواجه شوند إنداد

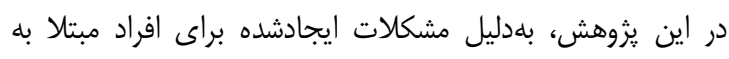

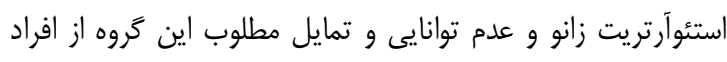

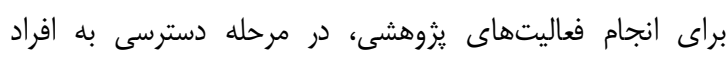

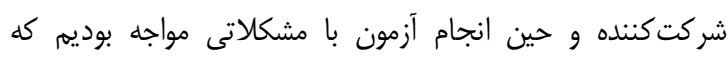

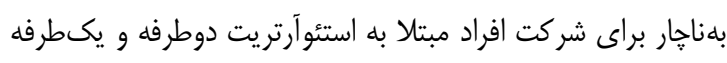

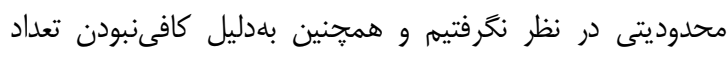

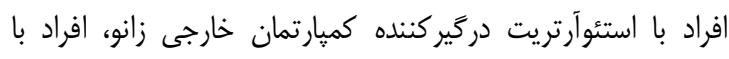

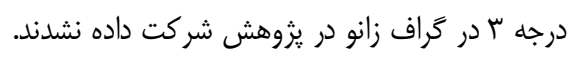

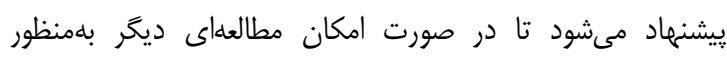

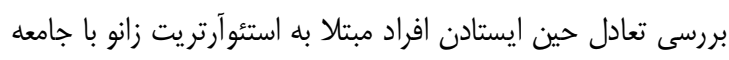

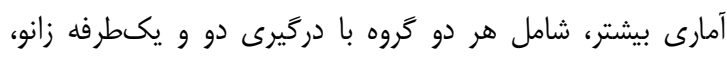

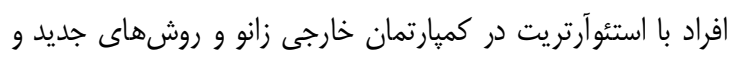

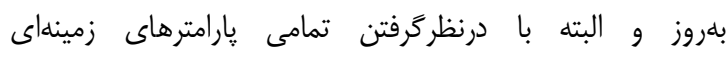

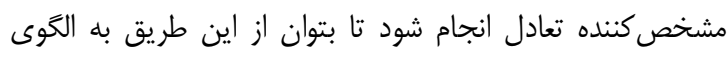

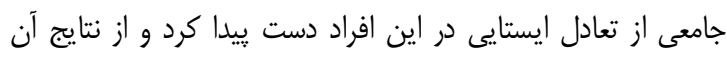

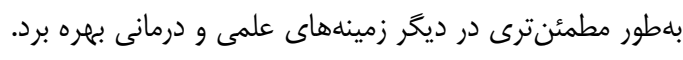

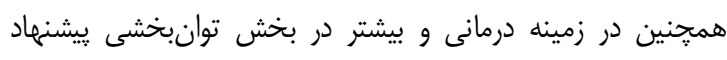

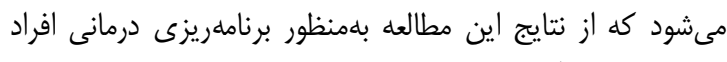

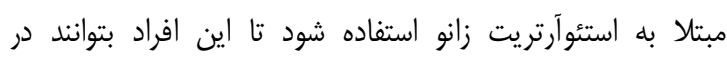

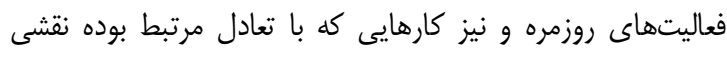

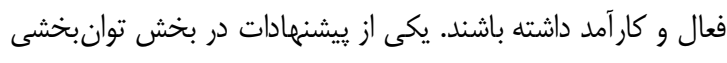

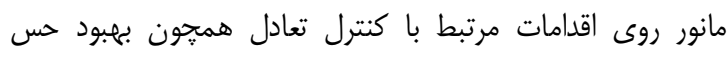

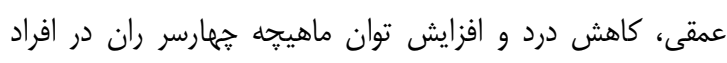

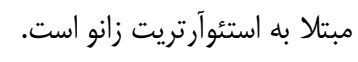

\section{نتيجه كَيرى}

ثبات حين ايستادن (ثبات ايستايى) در افراد مبتاديا به استئوآرتريت زانو نسبت به افراد سالم كاهش بيدا مى كند.

تشكر و قدردانى: از تمام شركت كنندكانى كه ما را در انجام اين

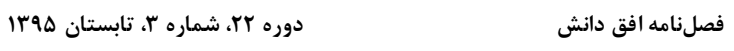


ثبات ايستادن در مبتلايان به استئو آرتريت زانو 199

22- Hsieh RL, Lee WC, Lo MT, Liao WC. Postural stability in patients with knee osteoarthritis: comparison with controls and evaluation of relationships between postural stability scores and International Classification of Functioning, Disability and Health components. Arch Phys Med Rehabil. 2013;94(2):340-6.

23- Cybulski GR, Jaeger RJ. Standing performance of persons with paraplegia. Arch Phys Med Rehabil. 1986;67(2):103-8.

24- Murray MP, Seireg AA, Sepic SB. Normal postural stability and steadiness: Quantitative assessment. J Bone Joint Surg Am. 1975;57(4):510-6.

25- Doyle TL, Newton RU, Burnett AF. Reliability of traditional and fractal dimension measures of quiet stance center of pressure in young, healthy people. Arch Phys Med Rehabil. 2005;86(10):2034-40.

26- Swanenburg J, de Bruin ED, Favero K, Uebelhart D, Mulder T. The reliability of postural balance measures in single and dual tasking in elderly fallers and non-fallers. Bio Med Central Musculoskelet Disord. 2008;9:162.

27- Lafond D, Corriveau H, Hebert R, Prince F. Intrasession reliability of center of pressure measures of postural steadiness in healthy elderly people. Arch Phys Med Rehabil. 2004;85(6):896-901.

28- Zhang Y, Jordan JM. Epidemiology of osteoarthritis. Clin Geriatr Med. 2010;26(3):355-69.

29- Felson DT, Zhang Y, Hannan MT, Naimark A, Weissman BN, Aliabadi $\mathrm{P}$, et al. The incidence and natural history of knee osteoarthritis in the elderly, the framingham osteoarthritis study. Arthritis Rheum. 1995;38(10):1500-5.
14- Pirayeh N, Talebian S, Hadian MR, Olyaei GR, Jalaei Sh, Mazaheri H. Assessment of balance impairments in patients with knee osteoarthritis. Mod Rehabil. 2010;4(3-4):18-22. [Persian]

15- Raymakers JA, Samson MM, Verhaar HJ. The assessment of body sway and the choice of the stability parameter(s). Gait Posture. 2005;21(1):48-58.

16- March LM, Bachmeier CJ. Economics of osteoarthritis: A global perspective. Baillieres Clin Rheumatol. 1997;11(4):817-34.

17- Nagaosa Y, Mateus M, Hassan B, Lanyon P, Doherty M. Development of a logically devised line drawing atlas for grading of knee osteoarthritis. Ann Rheum Dis. 2000;59(8):587-95.

18- Esrafilian A, Karimi MT, Amiri P, Fatoye F. Performance of subjects with knee osteoarthritis during walking: Differential parameters. Rheumatol Int. 2013;33(7):1753-61.

19- Davis MA, Ettinger WH, Neuhaus JM, Mallon KP. Knee osteoarthritis and physical functioning: Evidence from the NHANES I epidemiologic followup study. J Rheumatol. 1991;18(4):591-8.

20- McDaniel G, Renner JB, Sloane R, Kraus VB. Association of knee and ankle osteoarthritis with physical performance. Osteoarthritis Cartilage. 2011;19(6):634-8.

21- Birmingham TB, Kramer JF, Kirkley A, Inglis JT, Spaulding SJ, Vandervoort AA. Knee bracing for medial compartment osteoarthritis: effects on proprioception and postural control. Rheumatol. 2001;40(3):285-9. 